\title{
Application of contact elements to represent prismatic mechanical couplings
}

\author{
Mahdi Ghesmi*, and Bettar Ould el Moctar \\ Institute of Ship Technology, Ocean Engineering and Transport Systems, University of Duisburg- \\ Essen, 47048 Duisburg, Germany
}

\begin{abstract}
Accurate prediction of loads on mechanical couplings is crucial in assessment of loads on coupled structures and in optimization of mechanical coupling design. In this paper, a contact element model is introduced to represent prismatic joints. A twofold pushing convoy in shallow water was taken for time domain numerical simulations in regular waves. The prismatic joints interconnecting the convoy bodies allowed relative heave and pitch motions of the bodies relative to each other. The articulation forces and body motions were compared to model basin measurements to assess the reliability of contact element model. The contact element model could simulate the prismatic joints efficiently and it provided a suitable method to idealize free and suppressed modes at articulation locations.
\end{abstract}

\section{Introduction}

The insatiable and continuous demand for higher capacity ships has always been an advancing force driving many technological fields of naval architecture. Realization of container mega boxers with TEU (Twenty Equivalent Unit) capacities of more than 20000 is an example of such achievements. Not only the design and construction of higher capacity vessels, but also their operation is a technical challenge. One operational hindrance is that not all terminals are able to accommodate such vessels; thus, redesign of available terminals or planning new dedicated terminals are demanded. In the latter case, due to the high draft of large vessels, some terminals are forced to set up their facilities out of reach of inland vessels. France's Le Havre deep terminal and Germany's Jade-Weser port are two examples of such ports, where the inland vessels are territorially exposed to open sea conditions. Deep-sea terminals are connected to inland water infrastructure by so-called river-sea vessels, which are predominantly river vessels adapted for sea voyages. The adaption of river vessels to sail at sea requires special technical and safety measures. In the middle of the millennium's first decade, various national regulations associated with riversea vessels appeared. France and Belgium published their stability related regulations in 2007. France regulated the inland vessels traveling to the Le Havre deep sea terminal [1] and Belgium released prescriptive rules for the inland vessels traveling between

\footnotetext{
* Corresponding author: mahdi.ghesmi@uni-due.de
} 
Westerschelde and Belgian deep waters [2-4]. Following these rules, PIANC (Permanent International Association of Navigation Congresses) initiated a safety committee for such vessels, and UNECE (United Nations Economic Commission for Europe) issued the Recommendations on Harmonized Europe-Wide Technical Requirements for Inland Navigation Vessels [1].

Germany initiated a federally sponsored project in 2012 to study the linking options of Germany's deep sea Jade-Weser port to inland waterways by means of river-sea vessels. Especially for economic reasons, a river-sea pushing convoy consisting of a pushing tug and a pushed barge was chosen as the favorite solution. One of the major challenges investigated within the framework of this project was the determination of loads acting on the complex coupling mechanism of the convoy, which included prismatic joints.

Prismatic or translation joints allow relative translation between two bodies along the joint axis. The use of prismatic joints eliminates the freedom of a body to translate relative to the other body in any other direction except along the joint axis. It also eliminates the freedom of the body to rotate with respect to the other body [5]. Application of prismatic joints in coupling two or several ships and offshore structures is not very prevalent - in contrary to hinge joints. However, common methods used in ship and offshore field to model hinge joints can generally be used for prismatic couplings. There exits several different methods to model mechanically coupled multibody systems. Penalty methods replace the constraints imposed on the system by coupling mechanism by a series of unconstraint problems, which involve penalty functions [6]. The deviation of the evaluated function from zero is the measure of violation, against which a penalty force must withstand. Penalty methods are computationally inexpensive but the simulation quality is dependent on penalty function coefficients, which are not easy to determine. Use of penalty methods in ship and offshore hydrodynamics is still rare. One example can be found in [7]. Like penalty methods, Lagrange multiplier method uses forces to treat coupling constraints. However, in penalty methods these internal forces are exerted after determination of the violation measurements, but in Lagrange multiplier method the internal forces must avoid occurrence of such violations in compliance with defined constraints. Lagrange multiplier method is very common in ship and offshore industry. An applications of this method can be found in [8]. Penalty and Lagrange multiplier methods rely on determined internal forces to fulfill coupling constraints. The method of generalized coordinates follows principally a different approach and considers the effect of coupling on a system, i.e. constraints imposed on a system by a coupling reduces the degrees of freedom of that system. An example of application of generalized coordinate method can be found in [9]. Mode expansion method is probably the most widespread method in treating multibody systems in marine and offshore field. Probably Newman [10-16] has been the first who showed the versatility of this approach in association with boundary element fluid solvers. In mode expansion method, all degrees of freedom are described by mode shapes. Generalized modes can have any shapes and describe both rigid body and flexible modes. Therefore, this concept subsumes both seakeeping and interconnection theories in the sense that rigid or flexible bodies can be connected to other fixed or rigid bodies with rigid or flexible fixtures. The drawback of this method is that it relies on mode superposition principle, which necessitates linearity of the analyzed system. In [17-20] various implementations of this method in marine industry are presented. Recently, Ghesmi et. al [21] employed contact elements to represent hinge mechanical couplings. Application of contact elements eliminates the necessity to incorporate articulation constraints as in Lagrange multiplier and generalized coordinate methods and does not require system linearity as in mode expansion method. Forces induced by contact elements can be integrated directly as external forces into motion equations [22]. In the present paper, we extended the application of contact element approach introduced and validated in [21] to prismatic joints. The aforementioned 
river-sea convoy was taken for validation purposes. The hydrodynamic package Ansys Aqwa [23] was used to simulate the convoy in time domain. The predicted ship motions and loads on the mechanical connections were validated against model test measurements. Since these model test results have not been published in any scientific journals or conferences yet, a brief summary of the model tests was included in the validation section.

\section{Description of numerical method}

\subsection{Contact elements}

Contact elements defined herein are damper-spring massless elements. A contact element acts only in compression between a point on one structure and a contact plane on another. A point and a normal vector define the contact plane. The compression force $\vec{F}_{k}$ on the contact element acts in normal direction. Its magnitude $F_{k}$ is defined as a polynomial function of the compression $\Delta l$, i.e.

$$
F_{k}=\left\{\begin{array}{cc}
\sum_{i} C_{k i} \Delta l^{i} & \text { if } \Delta l>0 \\
0 & \text { otherwise }
\end{array}\right.
$$

where $C_{k i}$ are contact element constants. The nonlinearity of the contact force is helpful to represent physical characteristics of a contact element, such as a contact element hardly compressed after reaching a certain compression threshold. Contact damping force, $\vec{F}_{d}$, is applied in the opposite direction of the element compression force, $\vec{F}_{k}$, and is related to element compression velocity. Its magnitude reads:

$$
F_{d}=C_{d} C_{k} \frac{\mathrm{d} \Delta l}{\mathrm{~d} t}
$$

$C_{d}$ and $C_{k}$ are damping and stiffness coefficients, respectively. The magnitude of the friction force, $F_{f}$, between both bodies with friction coefficient $\mu$ is:

$$
F_{f}=\mu F_{k}
$$

Friction force, $\vec{F}_{f}$, acts in the opposite direction to the velocity. Subsequently, the total contact force reads:

$$
\vec{F}_{t}=\vec{F}_{k}+\vec{F}_{d}+\vec{F}_{f}
$$

\subsection{Solution of hydrodynamic problem}

Contact elements used here are in congruence to time domain boundary element method to solve seakeeping behavior of multibody system. Usual three dimensional boundary element methods for the prediction of motions and loads in waves assume an inviscid and incompressible fluid and an irrotational flow. Due to these assumptions, a velocity potential exists, which satisfies Laplace equation in the fluid domain (conservation of mass) and suitable boundary conditions on the domain boundaries. On the ship surface and on the free surface, the condition of no flow through the surface in normal direction applies; the same condition applies on the bottom in shallow water. Additionally, the pressure on the free surface shall be equal to atmospheric pressure. Far away from ships, the flow shall not be disturbed by their presence.

Since contact element forces are not harmonic with constant amplitudes, time domain simulations following [24] was applied. Therefore, the motion equation of a system in time domain after incorporation of contact element forces as additional external forces reads: 


$$
(\mathbf{M}+\mathbf{A}(\infty)) \overrightarrow{\dot{\eta}}+\int_{0}^{\mathrm{t}} \mathbf{K}(t-\tau) \overrightarrow{\dot{\eta}}(\tau) \mathrm{d} \tau+\mathbf{R} \vec{\eta}=-\vec{F}_{F K}-\vec{F}_{D}-\sum \vec{F}_{t}
$$

where $t$ and $\tau$ represent time; $\vec{\eta}$ is the vector of ship motions, including translations and rotations; $R \vec{\eta}, \vec{F}_{F K}$, and $\vec{F}_{D}$ are hydrostatic, Froude-Krylov, and diffraction forces, respectively; and $\mathbf{A}(\infty)$ is the added mass matrix at infinite frequency; and $\mathbf{M}$ the mass matrix.

Here, hydrostatic force, $\mathbf{R} \vec{\eta}$, and Froude-Krylov force, $\vec{F}_{F K}$, are calculated nonlinearly in each time step by accounting for the instantaneous wetted surface. Diffraction forces, $\vec{F}_{D}$, are assumed linear and are transposed from prerequisite frequency domain calculations:

$$
\begin{array}{ll}
\vec{F}_{D}=\iint_{S} p_{D} \vec{n} \mathrm{~d} S & \text { (for forces) } \\
\vec{F}_{D}=\iint_{S} p_{D}(\vec{r} \times \vec{n}) \mathrm{d} S & \text { (for moments) }
\end{array}
$$

where $\vec{r}$ denotes the distance vector, $\vec{n}$ the unit normal, $S$ the wetted surface, and $p_{D}=-\rho g z-\rho \partial \varphi_{D} / \partial t$ the linearized diffraction pressure force acting on the hull surface. Coordinate $z=0$ is located at the undisturbed mean water level, $g$ is earth acceleration, $\rho$ is water density, and $\varphi_{D}$ is the diffraction potential. The diffraction potential must satisfy the Laplace equation $\left(\Delta \varphi_{D}=0\right)$, the free surface condition $\left(\partial \varphi_{D} / \partial z-k \varphi_{D}=0\right)$, and the body boundary condition $\left(\partial \varphi_{D} / \partial n=0\right)$, where $k$ is the wave number. The impulse response function,

$$
\mathbf{K}(t)=\frac{2}{\pi} \int_{0}^{\infty} \mathbf{B}\left(\omega_{e}\right) \cos \omega_{e} t \mathrm{~d} \omega_{e}
$$

is computed from the frequency dependent linear damping matrix, $\mathbf{B}\left(\omega_{e}\right)$, where $\omega_{e}$ is the encounter frequency. Matrix $\mathbf{B}\left(\omega_{e}\right)$ is obtained from frequency domain radiation forces. Radiation forces are calculated similarly to diffraction forces, whereby the radiation potential, $\varphi_{R}$, must fulfill the body boundary condition $\left(\partial \varphi_{R} / \partial n=i \omega_{e} n\right)$. Knowing that $j^{\text {th }}$ radiation force is due to the radiation wave induced by $k^{\text {th }}$ unit amplitude body motion, it can be computed from

$$
F_{R j k}=\omega_{e}^{2} A_{j k}+i \omega_{e} B_{j k}
$$

where $A$ and $B$ are elements of added mass matrix $\mathbf{A}$ and damping matrix $\mathbf{B}$. Added mass at infinite frequency, $\mathbf{A}(\infty)$, is calculated by considering that the complex function

$$
\mathbf{A}\left(\omega_{e}\right)-\mathbf{A}(\infty)+\frac{i}{\omega_{e}} \mathbf{B}\left(\omega_{e}\right)
$$

is analytic in the upper half plane. Therefore, with $\mathbf{A}\left(\omega_{e}\right)$ and $\mathbf{B}\left(\omega_{e}\right)$ directly available from frequency domain calculations, an asymptotic added mass matrix at infinity $\mathbf{A}(\infty)$ can be obtained.

\section{Validation of numerical model}




\subsection{Test case}

The tests were conducted at the Development Centre for Ship Technology and Transport Systems (DST) in a shallow water tank of $200 \mathrm{~m}$ length, $10 \mathrm{~m}$ width, and $1.2 \mathrm{~m}$ depth. The shallow water basin was equipped with hydraulic wave makers, which were adjusted continuously between flap and piston mode. The adjustable water depth and the design of the wave makers allowed simulating various conditions, including shallow water effects on wave dynamics. The generated waves were absorbed by a fixed iron beach at the basin's end. The water depth was adjusted to represent $17 \mathrm{~m}$ full scale depth. The model tests with appendages were conducted at 1:20 scale (figure 1). Figure 2 and table 1 show body plans of the convoy bodies and their main particulars, respectively.

Table 1. Main particulars of convoy bodies (full scale).

\begin{tabular}{|c|c|c|c|c|c|c|c|}
\hline & $L[\mathrm{~m}]$ & $B[\mathrm{~m}]$ & $T[\mathrm{~m}]$ & {$\left[\mathrm{m}^{3}\right]$} & $C O G(x, y, z)[\mathrm{m}]$ & $G M[\mathrm{~m}]$ & $r(r x x, r y y, r z z)[\mathrm{m}]$ \\
\hline Barge & 90.0 & 11.4 & 2.5 & 2427 & $73.7,0,0.36$ & 2.7 & $2.78,23.1,23.1$ \\
\hline Tug & 27.8 & 8.8 & 3.0 & 402 & $14.8,0,-1.07$ & 2.7 & $3.6,7.5,7.5$ \\
\hline
\end{tabular}

$L, B, T, \nabla, C O G, G M, r, r_{x x}, r_{y y}, r_{z z}$ denote body's length, breadth, draft, displacement, center of gravity, metacentric height and radii of gyration (in $\mathrm{x}, \mathrm{y}$, and $\mathrm{z}$ directions), respectively.

Dedicated tests were run in regular waves to validate numerical simulations. The tests were conducted in head, beam, and following waves at zero forward speed. The bodies were positioned with moorings. A hawser was fastened tightly across the tank, to which the barge's forward end in head waves and the tug's aft end in following waves were connected by means of spring links. The other end of the convoy body was fastened to an elastic hawser, which was also drawn across the tank (figure 4). In beam waves, two spring links connected the barge to the taut hawser.

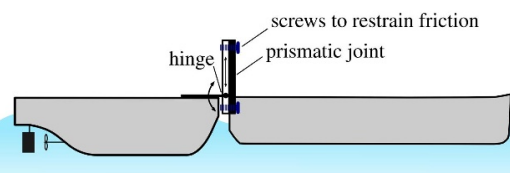

Fig. 1. Arrangement of model test setup. The tests were performed for the convoy at zero forward speed and for various regular wave angles of attack.

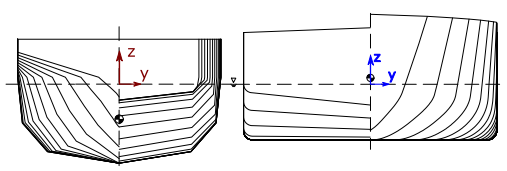

Fig. 2. Body plans of the convoy's tug (left) and barge (right).

The modeled coupling consisted of several components, with each component fulfilling one specific purpose (figure 3). The relative heave motion was realized by a prismatic joint, but restricted in its extremities by intervening mechanicals stops. The freedom of rotation of both vessels was ensured by a hinge at the point where the tug was connected to the prismatic connectors. Arbitrary pretension could be modeled by governing the friction along the prismatic joint axis via screws (figure 1). Screws were so adjusted to impose 80 $\mathrm{kN}$ pretenstion in all test runs. Relative surge and sway motions in combination with the application of friction force were considered suppressed. Relative roll motion characteristics of such a configuration were influenced by the system's geometric configuration and by the friction force. 


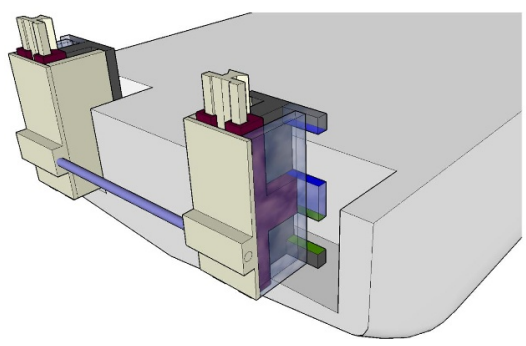

Fig. 3. Coupling mechanism employed in model tests.

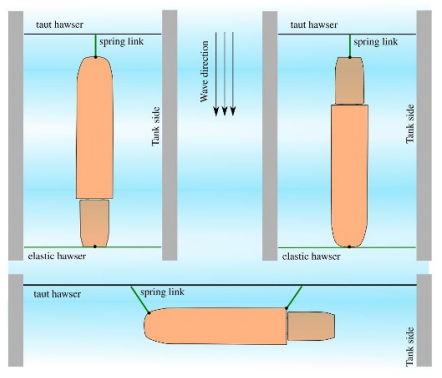

Fig. 4. Test arrangements for runs in regular waves.

\subsection{Convoy in regular beam waves}

In lieu of attaching the barge with two spring links to a taut hawser, barge sway and yaw motions were suppressed. Other barge motions and all tug motions were free. Mechanical coupling between vessels were modeled by control elements. Pretention was applied in accordance with model tests, i.e. as friction forces. Relative vertical motion was restrained by friction along prismatic joint axes. Friction force is direction-dependent and required assessment of relative motion of bodies at each time step.

Figure 5 shows the resultant roll and force responses obtained from numerical simulations and measurements. The numerical model predicted the force and motion responses relatively well in long waves, but the predictions deviate with decreasing wave lengths, with the tendency to underpredict the responses. Simulations predicted extremely high roll motions for the $1.47 \mathrm{rad} / \mathrm{s}$ frequency. Studying the almost linear course of roll responses between 0.84 and $1.47 \mathrm{rad} / \mathrm{s}$ reveals that the convoy was prevented from rolling more than about $6.5 \mathrm{deg} / \mathrm{m}$ because of attached spring links.
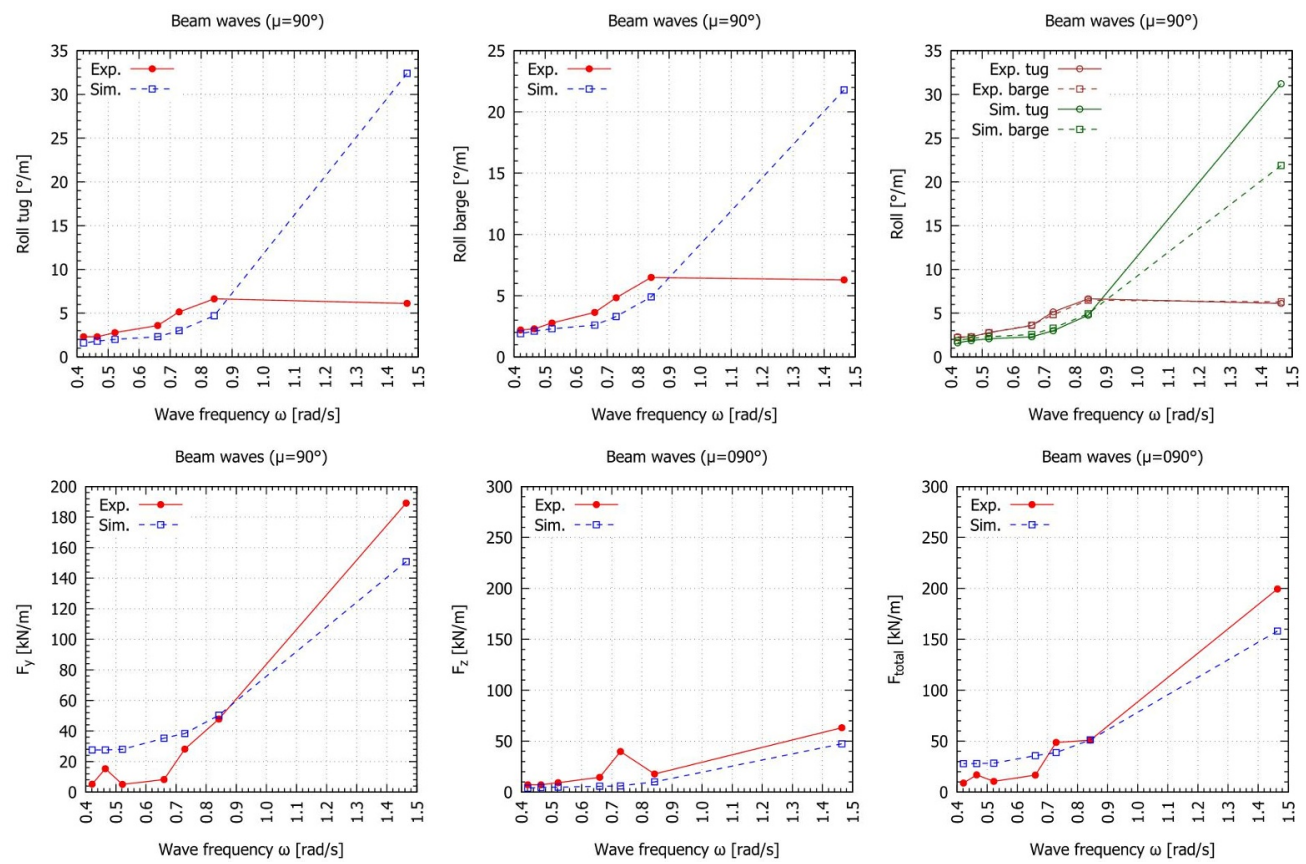

Fig. 5. Roll motions of convoy bodies per wave amplitude (top) and coupling forces per wave amplitude (bottom) in beam waves. 
The transverse component of total force response was generally overestimated by numerical model for all examined wave frequencies, except for $\omega=0.84$ and $1.47 \mathrm{rad} / \mathrm{s}$. On the other hand, all vertical force components were slightly underestimated. This caused the calculated total forces, consisting of transverse and vertical components, to agree favorably with measurements. The two bumps at 0.47 and $0.74 \mathrm{rad} / \mathrm{s}$ for vertical and transverse coupling forces could not be captured in simulations. Taking the relatively smooth track of roll responses into account, it is believed that these two bumps were related to inaccuracies in measurements or their respective time series assessments. Nevertheless, further measurements for these two wave frequencies, and preferably also for wave frequencies in their vicinities, are required before making any final statements related to the bumps. Relatively extreme forces were found for $1.47 \mathrm{rad} / \mathrm{s}$. Although the predicted vertical components at $1.47 \mathrm{rad} / \mathrm{s}$ still agree, the transverse component underpredicted the measurements unacceptably. Considering that the spring links prevented the vessels from rolling further than a threshold, the link forces were transmitted to the system, and this was reflected accordingly in the resultant coupling forces. This is why the discrepancies of measurements and simulations originated from different configurations. These differences are believed to have had fewer effects on the results at $\omega<=0.84 \mathrm{rad} / \mathrm{s}$, probably because of reduced effects of spring links on the responses. Subsequently, we concluded that the responses for $1.47 \mathrm{rad} / \mathrm{s}$ were not considered valid, neither for simulations nor measurements.

Figure 6 shows time series of coupling forces for $\omega=0.66 \mathrm{rad} / \mathrm{s}$ in waves of $9.5 \mathrm{~s}$ period and $140 \mathrm{~m}$ length. Attention is drawn to measured time series of vertical forces and the existence of excessive local extrema, which impeded the analyses of time series and were a source of departure of measurements from simulations. Altogether, numerical model could predict the measured forces well. Figure 7 shows time series of relative vertical motion of both bodies at port and starboard couplings. The continuous green line indicates the higher position of the tug against the barge at the coupling position. The dotted blue peaks imply the lagging tug movement, such that the barge moves first, engages the tug, and drags it along with it. Figure 7 shows negligibly small relative vertical motion of bodies along the axes of prismatic joints.
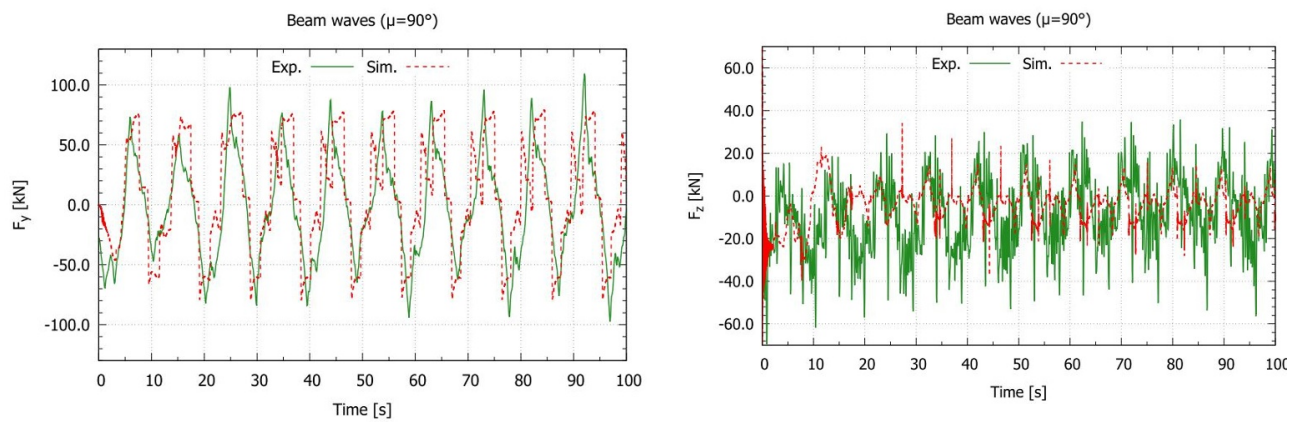

Fig. 6. Time series of coupling forces in transverse (left) and vertical (right) directions. Tests were conducted in beam waves of $9.5 \mathrm{~s}$ of period and $140 \mathrm{~m}$ of length.

After studying the tug and barge roll motions shown in the top right graph of figure 5, it becomes evident that both bodies rolled almost synchronously. It originated from the fact that the vertical motions of the bodies, in absence of pitch motion, consisted of heave and roll vertical components. Due to small relative vertical motion of the bodies, roll responses of both bodies became nearly equal. Consequently, it was concluded preliminarily that beam wave cases might also be simulated ideally with hinges, because no relative vertical motion are allowed by hinge joints. 

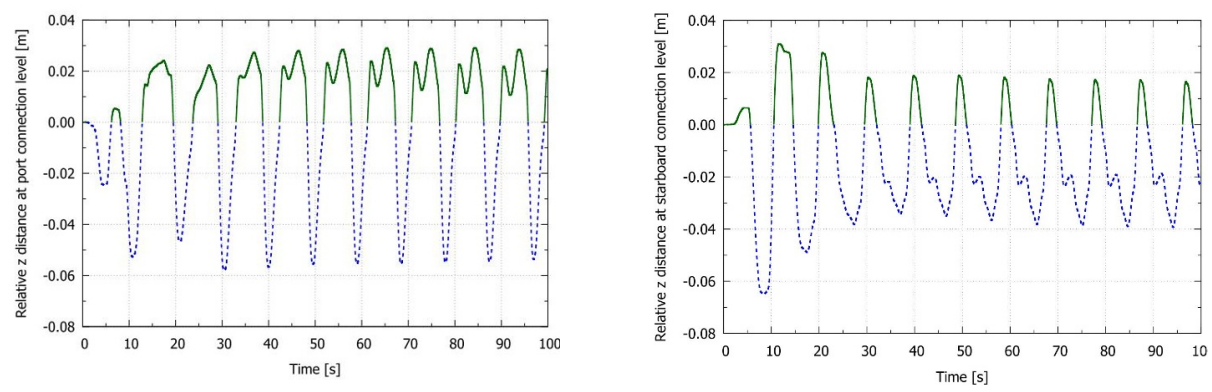

Fig. 7. Time series of relative vertical distance between bodies at port (left) and starboard (right) connections. Tests were conducted in beam waves of $9.5 \mathrm{~s}$ of period and $140 \mathrm{~m}$ of length.

\subsection{Convoy in regular head and following waves}

Similar to beam wave simulations, no moorings were involved in following wave simulations and we preferred suppressing degrees of freedom. The tug's surge and yaw modes were suppressed, and all other barge and tug degrees of freedom were free. Again, the effect of spring links was neglected.

Figure 9 shows pitch motions of the bodies per wave amplitude. Simulations predicted the pitch motions fairly well, especially pitch motions of the barge. The relatively less favorable pitch prediction of the tug is ascribed to tug's sensibility to external forces. The sudden rise of barge's simulated pitch at $\omega=0.47 \mathrm{rad} / \mathrm{s}$ is believed to be due to simulation inaccuracies because neither force predictions and measurements, nor tug's motion predictions and measurements registered such a rise. The frequency of $\omega=0.52 \mathrm{rad} / \mathrm{s}$ is among the wave frequencies for which the prismatic joints were believed to be more wetted (or less tensed) because measurements could not be reconstructed unless about $75 \%$ of contact element forces for $\omega<0.52 \mathrm{rad} / \mathrm{s}$ simulations were applied. Analogous to beam waves, the vertical component of reaction forces tended to underestimate the responses, and the horizontal components tended to overestimate them; hence, the reaction force comprised these two components led to satisfactory prediction of resulting forces. In following waves, $80 \mathrm{kN}$ pretension force at each connection proved not to be high enough to keep both vessels attached at initial coupling locations. The left graph in figure 8 shows great relative vertical motion of the bodies. Comparing both graphs in figure 8 reveals not only the exposure and shielding characteristics of the tug in head and following waves, but also the effects of pretension.
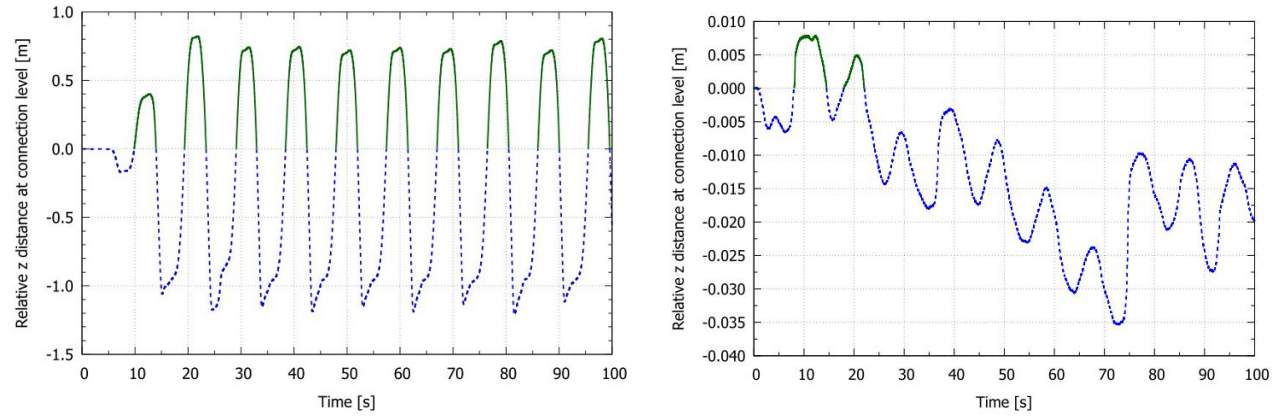

Fig. 8. Time series of relative vertical distance between bodies at port and starboard connections.

Tests were conducted in following (left) and head (right) waves of $9.5 \mathrm{~s}$ of period and $140 \mathrm{~m}$ of length. 

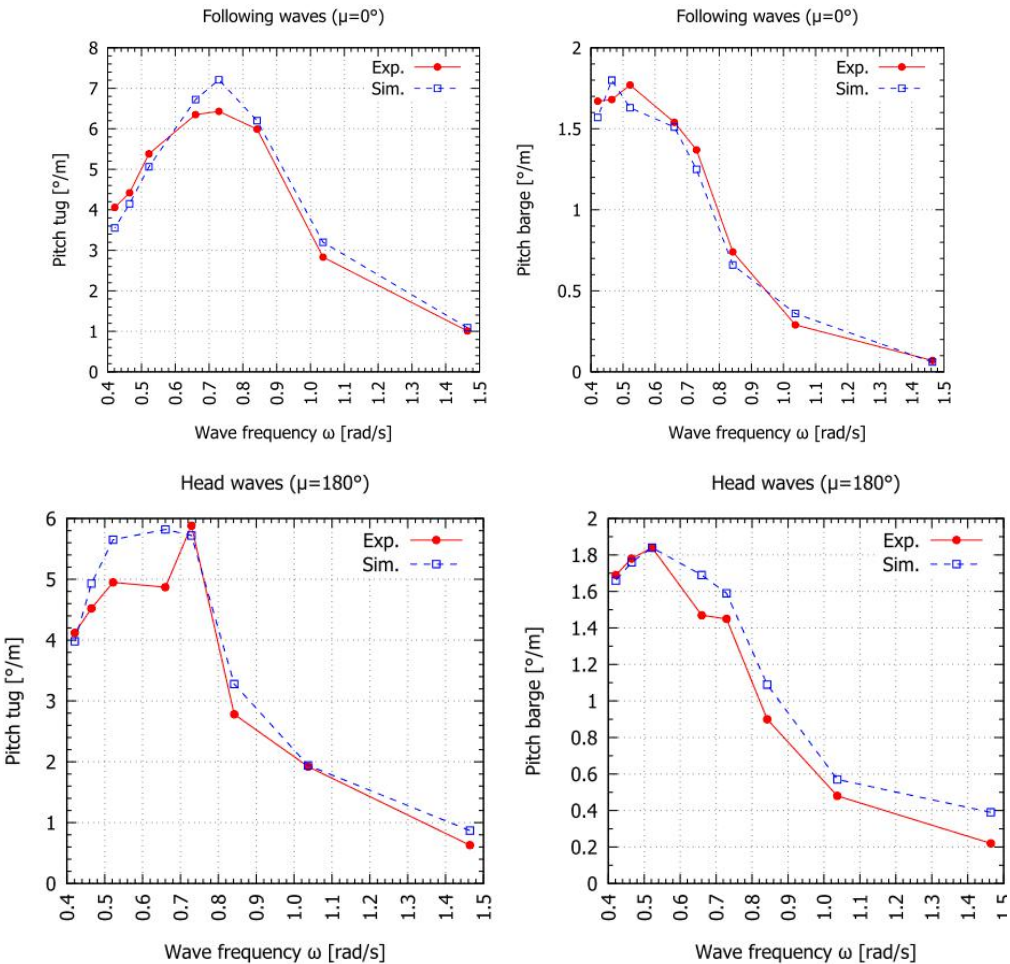

Fig. 9. Pitch motions per wave amplitude in following (top) and head (bottom) waves.
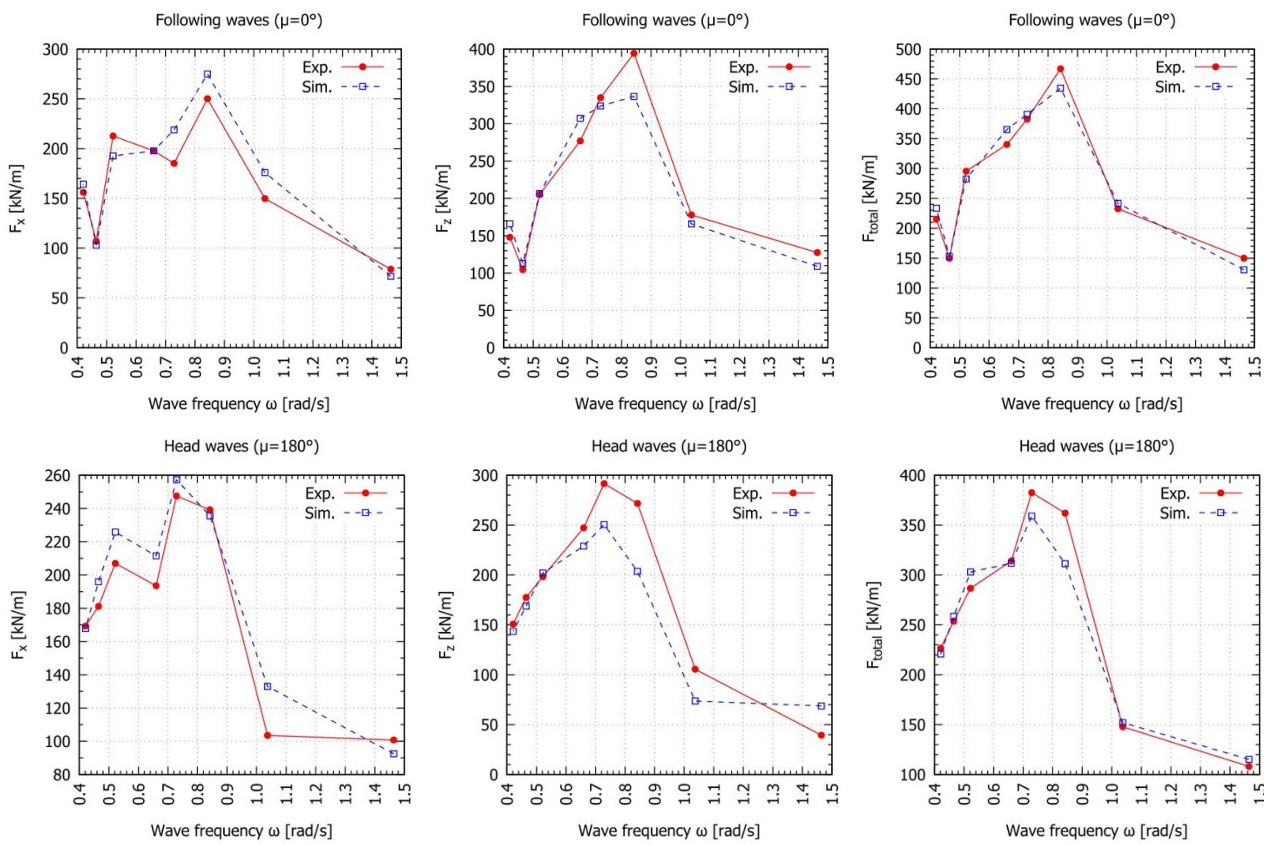

Fig. 10. Coupling forces per wave amplitude in following (top) and head (bottom) waves.

Simulations in head waves were generally similar to those in following waves i.e. instead of spring links, suppression of appropriate degrees of freedom of the barge was 
imposed. Similar to model tests with following waves, relative motions of bodies at both port and starboard couplings were the same. Relative vertical distances at connection positions, as seen in the right graph of figure 8, are negligible in comparison with the following wave counterparts. Figure 9 and 10 show motion and force responses, respectively. As already experienced, simulations provided smooth motion response amplitude operator curves; however, the corresponding experimental curves showed pitfalls or bumps. The abrupt pitfalls in measurement pitch curves were not tracked by computations. Recurrently, overestimation of horizontal forces and underestimation of vertical forces were observed, which led to satisfactory prediction of total resultant forces. Altogether, contact element models predicted the force response traces fairly well, except for $\omega>1.04 \mathrm{rad} / \mathrm{s}$. The reason for this inaccuracy might have been the increase of nonlinearities with decreasing wave lengths and the behavior of the coupling in short waves - in short waves friction force changed more intensively and the contact element model must come along with frequent friction force changes.

\subsection{Effect of pretension}

After validation of contact element model in representing prismatic joints, we examined the efficiency of pretension. The contact element model provided a qualified method to comfortably conduct parametric studies on pretension efficiency. To assess the effect of pretension, relative vertical motion between bodies was assessed. As this motion was generally more prominent in following waves, following waves with an amplitude of 1.22 $\mathrm{m}$ and a frequency of $0.74 \mathrm{rad} / \mathrm{s}$, which corresponded to waves of $7.5 \mathrm{~s}$ period and of $87 \mathrm{~m}$ length, were chosen. Exposed to these waves, the convoy underwent severe relative motions with hard impacts on prismatic joint ends. Figure 11 shows the time series of relative vertical motions at couplings. Due to absence of roll motion, both starboard and port couplings registered identical time series. The influence of pretension was evident as the relative vertical motion mitigated with increasing pretension magnitude. How effective the pretension decreased the relative vertical motion, i.e., how intensive the pretension kept both bodies attached, was quantified by measuring the distance between maximum and minimum peak values, i.e., the relative vertical distance. Figure 11 shows that increasing pretension did not decrease the relative vertical distance linearly. We performed regression studies based on linear, exponential, and second order polynomial functions. These showed that the two latter functions predicted satisfactorily the correlation between pretension and relative vertical distance (figure 12).

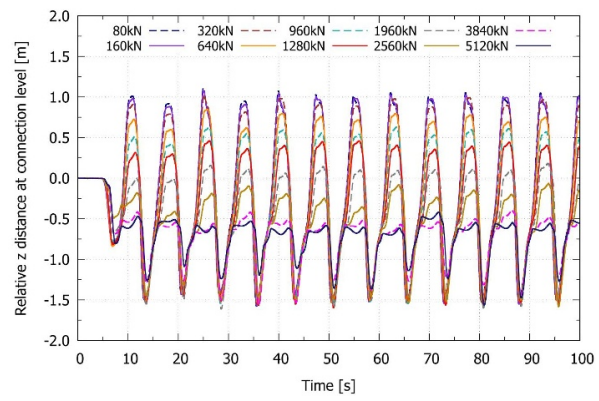

Fig. 11. Time series of relative vertical distance between bodies at connections level for different pretensions. Tests were conducted in following waves of $7.5 \mathrm{~s}$ of period and $87 \mathrm{~m}$ of length.

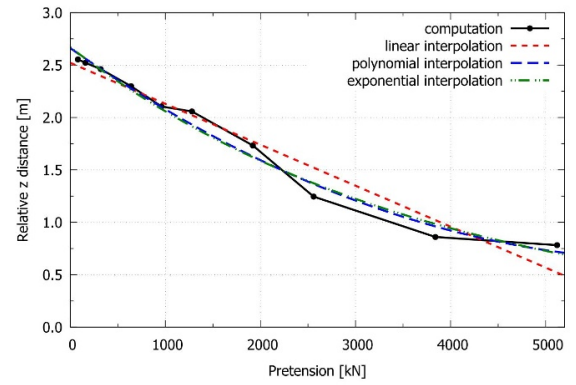

Fig. 12. Relative vertical distance between peaks of corresponding time series in Figure 12 vs. pretension. Tests were conducted in following waves of $7.5 \mathrm{~s}$ of period and $87 \mathrm{~m}$ of length. 


\subsection{Nonlinear effects}

To examine the influence of nonlinear Froude-Krylov and hydrostatic forces on articulation forces and body motions, we multiplied the wave amplitude by the factors 1.3, 1.5, and 2.0. Theoretically, more severe nonlinear affects were expected in steeper waves. For a wave frequency of $1.46 \mathrm{rad} / \mathrm{s}$, the highest amplification factor was 1.8 to avoid wave breaking. Table 2 shows wave angular frequencies, wave amplitudes, and wave steepnesses we considered.

Figure 13 shows the resulting pitch motions of the tug and vertical forces on couplings in waves of different amplitudes obtained from linear and nonlinear solutions in head waves. Model test measurements are shown for comparison. Nonlinear effects can be identified for almost all frequencies. Generally, nonlinear effects improved the response predictions. However, these effects increased also the inaccuracies of contact element model in prediction of vertical coupling forces at high wave frequencies.

Head waves $\left(\mu=180^{\circ}\right)$

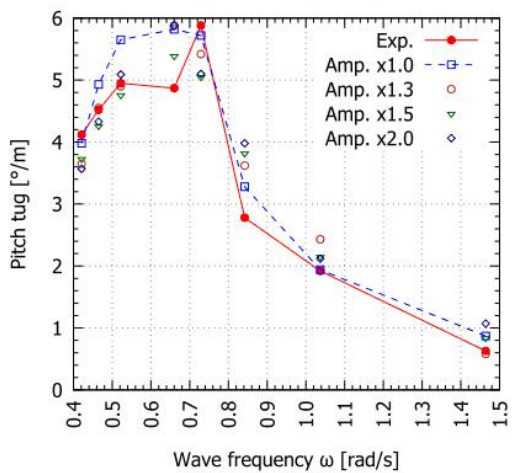

Head waves $\left(\mu=180^{\circ}\right)$

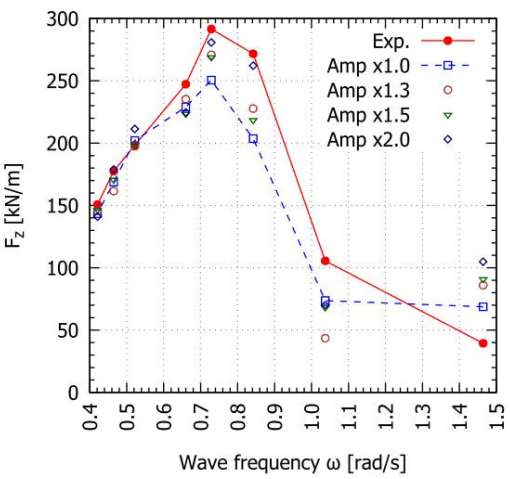

Fig. 13. Effect of nonlinear Froude-Krylov and hydrostatic forces on tug's pitch and coupling vertical force responses in head waves of different amplitudes and amplification factors.

Table 2. A Wave angular frequencies, amplitudes, and steepnesses.

\begin{tabular}{|c|c|c|c|c|c|c|c|c|c|}
\hline $\begin{array}{c}\omega \\
{[\mathrm{rad} /} \\
\mathrm{s}]\end{array}$ & $\begin{array}{c}\lambda \\
{[\mathrm{m}]}\end{array}$ & $\begin{array}{c}h \\
{[\mathrm{~m}]}\end{array}$ & $\begin{array}{c}100 \\
H / \lambda \\
{[-]}\end{array}$ & $\begin{array}{c}1.3 h \\
{[\mathrm{~m}]}\end{array}$ & $\begin{array}{c}100(1.3 \\
H / \lambda) \\
{[-]}\end{array}$ & $\begin{array}{c}1.5 h \\
{[\mathrm{~m}]}\end{array}$ & $\begin{array}{c}100(1.5 \\
H / \lambda)[ \\
-]\end{array}$ & $\begin{array}{c}2.0 h \\
{[\mathrm{~m}]}\end{array}$ & $\begin{array}{c}100(2.0 \\
H / \lambda) \\
{[-]}\end{array}$ \\
\hline 1.46 & 28.76 & 1.12 & 7.79 & 1.46 & 10.13 & 1.68 & 11.68 & $2.02 *$ & $14.02^{*}$ \\
\hline 1.04 & 57.32 & 1.05 & 3.66 & 1.37 & 4.76 & 1.58 & 5.50 & 2.10 & 7.33 \\
\hline 0.84 & 86.94 & 1.22 & 2.81 & 1.59 & 3.65 & 1.83 & 4.21 & 2.44 & 5.61 \\
\hline 0.73 & 115.98 & 1.15 & 1.98 & 1.50 & 2.58 & 1.73 & 2.97 & 2.30 & 3.97 \\
\hline 0.66 & 141.50 & 1.22 & 1.72 & 1.59 & 2.24 & 1.83 & 2.59 & 2.44 & 3.45 \\
\hline 0.52 & 226.21 & 1.09 & 0.96 & 1.42 & 1.25 & 1.64 & 1.45 & 2.18 & 1.93 \\
\hline 0.47 & 285.06 & 1.14 & 0.80 & 1.48 & 1.04 & 1.71 & 1.20 & 2.28 & 1.60 \\
\hline 0.42 & 347.76 & 1.2 & 0.69 & 1.56 & 0.90 & 1.80 & 1.04 & 2.40 & 1.38 \\
\hline 1.04 & 57.32 & 1.05 & 3.66 & 1.37 & 4.76 & 1.58 & 5.50 & 2.10 & 7.33 \\
\hline 0.84 & 86.94 & 1.22 & 2.81 & 1.59 & 3.65 & 1.83 & 4.21 & 2.44 & 5.61 \\
\hline
\end{tabular}

*Amplification factor 1.8 used instead.

Here $\omega, \lambda, h, H$ and $H / \lambda$ denote wave frequency, wave length, wave amplitude, wave height, and wave steepness, respectively 
Efficient and sufficiently accurate prediction of motions of coupled bodies and loads on mechanical couplings is an important task in multiple applications. Recently, Ghesmi et al (2018) used contact elements to model hinge joints. This paper extended the application of contact elements for prismatic joints. For validation purposes a convoy consisting of a pushed barge and a pushing tug coupled by a coupling mechanism was taken. The coupling mechanism composed prismatic joints, which allowed free heave and pitch motions of the bodies, and provided pretension capabilities to mitigate relative motions. Body motions and coupling forces in regular beam, head, and following waves were studied and compared to model test measurements. The study showed that contact element model was capable of predicting body motions and coupling loads accurately. However, the model provided inaccurate prediction of coupling loads at high wave frequencies. The contact element model was additionally used to study the effect of pretension on convoy motion behavior. The study showed that pretension mitigated the relative vertical motions between bodies exponentially. The pretension proved to be most effective in following waves, where relatively high relative motions originated from tug's susceptibility to external forces were arisen. Consequently, this paper demonstrated the suitability of contact elements in reliable representation of articulation mechanisms.

\section{Acknowledgments}

The project is funded by the German Federal Ministry of Economics and Technology (BMWi, grant No. 03SX359), whose support is acknowledged.

\section{References}

1. Bačkalov I 2012 A Probabilistic Analysis of Stability Regulations for River-Sea Ships. Proceedings of the 11th International Conference on the Stability of Ships and Ocean Vehicles

2. Truijens $P$ and Vantorre $M$ and Vanderwerff T 2006 On the design of ships for estuary service International Journal of Maritime Engineering vol 148 pp 1-15

3. Vantorre M and Eloot K and Delefortrie G 2010 Estuary Traffic: an Alternative Hinterland Connection for Coastal Ports Port Infrastructure Seminar 2010

4. Vantorre M and Eloot K and Delefortrie G 2012 Probabilistic Regulation for Inland Vessels Operating at Sea as an Alternative Hinterland Connection for Coastal Harbours. EJTIR pp 111-131

5. Shabana A A 2013 Dynamics of multibody systems (Cambridge university press)

6. Garcia de Jalon J and Bayo E 1994 Kinematic and dynamic simulation of multibody systems (Springer-Verlag)

7. Kim B W and Kyoung J H and Hong S Y and Cho S K 2005 Investigation of the effect of stiffness distribution and structure shape on hydroelastic responses of very large floating structures The Fifteenth International Offshore and Polar Engineering Conference

8. Guha A and Somayajula A and Falzarano J 2013 Analysis of Causeway Ferry Dynamics for Safe Operation of Improved Navy Lighterage System Proceedings of the 13th International Ship Stability Workshop

9. Xing-Kaeding Y 2006 Unified approach to ship seakeeping and maneuvering by a RANSE method PhD Thesis (Technical University Hamburg-Harburg) 
10. Newman J N 1994 Wave effects on deformable bodies Applied Ocean Research vol 16 pp 47-59.

11. Newman J N 1997 Wave Effects on Hinged Bodies Part I - Body Motions http://www.wamit.com/publications.htm

12. Newman J N 1997 Wave Effects on Hinged Bodies Part II - Hinge Loads http://www.wamit.com/publications.htm

13. Newman J N 1998 Wave Effects on Hinged Bodies Part III - Hinge Loads vs. Number of Modules http://www.wamit.com/publications.htm

14. Newman J N 1998 Wave Effects on Hinged Bodies Part IV - Vertical Bending Modes http://www.wamit.com/publications.htm

15. Newman J N 2001 Wave effects on multiple bodies Hydrodynamics in ship and ocean engineering vol 3 pp 3-26

16. Lee $\mathrm{C} \mathrm{H}$ and Newman $\mathrm{J} 2000$ An assessment of hydroelasticity for very large hinged vessels Proceedings of the second Int'l Conference on Hydroelasticity in Marine Technology, vol 14 pp 957-970

17. Fu S and Moan T and Chen X and Cui W 2007 Hydroelastic analysis of flexible floating interconnected structures. Ocean engineering vol 34 pp 1516-1531

18. Kim B W and Hong S Y and Kyoung J H and Cho S K 2007 Evaluation of bending moments and shear forces at unit connections of very large floating structures using hydroelastic and rigid body analyses. Ocean engineering vol 34 pp 1668-1679

19. Kim K H and Kim Y and Kim M S 2008 Time-domain analysis of motion responses of adjacent multiple floating bodies in waves In The Eighteenth International Offshore and Polar Engineering Conference

20. Tuitman J T and Malenica Š and Van't Veer R 2012 Generalized modes in timedomain seakeeping calculations Journal of Ship Research vol 56 pp 215-233

21. Ghesmi M and von Graefe A and Friedhoff B and Shigunov V and el Moctar B O 2018 Comparison and Validation of Numerical Methods to Assess Hydrodynamic Loads on Mechanical Coupling of Multiple Bodies Ship Technology Research, Schiffstechnik, https://doi.org/10.1080/09377255.2018.1482100

22. Fontijn H L 1988 Fender forces in ship berthing PhD Thesis (TU Delft)

23. Ansys Inc 2017 Ansys theory manual

24. Cummins W 1962 The impulse response function and ship motions. Schiffstechnik 9, p 101-109 